livraisons

d'Histoire

de l'Architecture

\section{Livraisons de l'histoire de l'architecture}

$38 \mid 2019$

Varia III

\title{
La reconstruction de la Première Guerre mondiale dans la photographie : l'exemple du Nord et du Pas- de-Calais
}

The reconstruction of the First World War in the photography: the example of the Nord and the Pas-de-Calais region

Der Wiederaufbau nach dem Ersten Weltkrieg in der Photographie. Das Beispiel

der Region Nord-Pas-de-Calais

\section{Angéline Nison}

\section{OpenEdition}

Journals

Édition électronique

URL : http://journals.openedition.org/lha/1517

DOI : 10.4000/lha.1517

ISSN : 1960-5994

Éditeur

Association Livraisons d'histoire de l'architecture - LHA

Édition imprimée

Date de publication : 15 décembre 2019

Pagination : 103-110

ISSN : 1627-4970

Référence électronique

Angéline Nison, «La reconstruction de la Première Guerre mondiale dans la photographie : l'exemple du Nord et du Pas-de-Calais », Livraisons de l'histoire de l'architecture [En ligne], 38 | 2019, mis en ligne le 02 janvier 2021, consulté le 05 février 2021. URL : http://journals.openedition.org//ha/1517 ; DOI : https://doi.org/10.4000//ha.1517 


\section{LA RECONSTRUCTION DE LA PREMIËRE GUERRE MONDIALE DANS LA PHOTOGRAPHIE : L'EXEMPLE DU NORD ET DU PAS-DE-CALAIS}

Les prémices de la photographie remontent à la Renaissance : afin de régler les questions de perspective, les peintres de l'époque recouraient au principe de la Camera Obscura ${ }^{1}$. Servant d'abord d'aide au tracé du dessin, elle fut utilisée par Thomas Wedgwood ${ }^{2}$ pour tenter de fixer l'image formée.

La photographie naît suite à de nombreuses recherches complémentaires, réalisées simultanément dans le domaine de la chimie et de l'optique. C'est au cours du XVIII ${ }^{\mathrm{e}}$ siècle, tandis qu'Heinrich Schultze, savant allemand, découvre que les sels d'argent réagissent à la lumière, J. Charles, scientifique français obtient des images en silhouettes sur un papier sensibilisé aux sels d'argent. Nous sommes alors en 1780 , et le concept de la photographie, c'est-à-dire une image dessinée par la lumière, est bel et bien né.

Au début des années 1820, Nicéphore Niépce, ingénieur français, se lance dans des recherches sur la fixation d'une image. Après de nombreuses tentatives, il parvient à fixer une image sur une plaque d'étain sensibilisée au bitume de Judée ${ }^{3}$. Il lui a fallu plus de 8 heures d'exposition pour réussir à obtenir la vue prise de la fenêtre de son grenier.

En 1829, il explique son invention dans la Notice sur l'Héliographie: "La découverte que j'ai faite et que je désigne sous le nom d'héliographie consiste à reproduire spontanément par l'action de la lumière, avec les dégradations de teintes du noir au blanc, les images reçues dans la chambre-obscure " ${ }^{4}$.

Suite au décès de Niépce, Louis Daguerre reprit ses recherches et inventa le daguerréotype. Au même moment, William Henry Fox Talbot présente ses « dessins photogéniques ", prémices des calotypes ${ }^{5}$.

1. La Camera Obscura est le nom donné à la forme la plus simple de la chambre noire : une pièce obscure avec une petite ouverture sur l'un de ses murs. L'image des objets éclairés de l'extérieur se forme sur le mur opposé. La Camera Obscura, mentionné par Aristote, au IV siècle avant J.-C., fut perfectionnée à l'aube du XVI ${ }^{\mathrm{e}}$ siècle par l'adjonction d'une lentille convergente.

2. Scientifique anglais.

3. Le bitume de Judée est une substance noire qui a la propriété de blanchir et de devenir insoluble aux endroits où la lumière s'est posée. Les peintres ont également eu recours à cette substance pour donner de la profondeur à leur tableau.

4. Anne Cartier-Bresson, Le Vocabulaire technique de la photographie, Paris, Marval, 2008, p. 30.

5. Voir l'ouvrage de Claude Nori, La photographie française des origines à nos jours, Paris, Contrejour, 1978, 159 p. 


\section{Les débuts de la photographie dans la région Nord-Pas-de-Calais}

Au cours des années 1820-1830, la vie culturelle et artistique du territoire de la région Nord-Pas-de-Calais connaît un essor considérable. Prenant appui sur le modèle parisien, de nombreuses associations voient le jour, notamment à Lille ou encore Valenciennes ${ }^{6}$. Des expositions et des salons sont organisés afin de faire connaître, voire reconnaître le travail des artistes régionaux. Certains peintres, amateurs de photographie, font le lien dans la région, entre photographie et peinture, notamment le groupe dit d'Arras, concepteur du cliché verre ${ }^{7}$ avec Camille Corot dans les années 1850 . Un procédé qui retiendra un moment l'attention de peintres tels qu'Eugène Delacroix ${ }^{8}$.

Si certains s'en désintéressent rapidement, d'autres vont jouer un rôle important dans le domaine de la photographie, comme Louis-Désiré Blanquart-Évrard, resté dans l'histoire comme l'un des piliers de l'histoire de la photographie?

Ce dynamisme artistique est étroitement lié aux profondes mutations que connaît la région. Dès 1820, l'industrie subit de multiples modifications. Le territoire s'urbanise, se transforme. Un nouveau paysage se construit avec le développement des usines et l'accroissement des villes.

C'est également l'époque où la découverte de Niépce et de Daguerre est rendue publique par le savant François Arago et devient fédératrice d'un véritable engouement pour les nouvelles images et représentations. La photographie, symbole de modernité, est le support d'une réalité qui met en avant la puissance de l'expansion industrielle.

La presse locale met en exergue le daguerréotype. Dès lors, l'invention envahit l'espace public. Les premières expositions et démonstrations se multiplient suivies par la commercialisation du procédé. Suscitant l'intérêt et la curiosité, le daguerréotype demeure malgré tout un objet coûteux dont la réalisation technique reste très contraignante. Toutefois, des améliorations, réduisant le temps de pose ainsi que le format du matériel, rendent le daguerréotype plus accessible. Ces derniers perfectionnements, associés à l'intérêt croissant pour l'image argentique, sont les éléments moteurs qui ont permis d'offrir une place pérenne à la photographie dans la région ${ }^{10}$.

6. Évelyne-Dorothée Allemand, Katia Baudin, Damien Camus, et al., 160 ans de photographie en NordPas-de-Calais, Roubaix, Association des conservateurs des musées du Nord-Pas-de-Calais, Actes Sud, 2001, p. 10.

7. Il s'agit de prendre une plaque de verre recouverte d'une substance sensible qui va noircir à la lumière. Une fois insolée, la plaque devient une matrice, celle-ci peut être gravée. Il suffit donc de dessiner en grattant la surface. Le dessin obtenu peut être tiré comme un négatif verre.

8. Évelyne-Dorothée Allemand, Katia Baudin, Damien Camus, et al., 160 ans de photographie..., ibid., p. 29.

9. Louis-Désiré Blanquart-Évrard (1802-1872) est un chimiste lillois qui a amélioré le procédé du calotype mais a surtout créé la première imprimerie photographique.

10. Évelyne-Dorothée Allemand, Katia Baudin, Damien Camus, et al., 160 ans de photographie..., ibid., p. 11. 
Dans les années 1840, la ville de Lille voit l'ouverture de studios photographiques, comme celui d'Alphonse Le Blondel ${ }^{11}$. Il inaugure une nouvelle ère, celle de la photographie professionnelle. La particularité de son studio lillois tient au fait que, contrairement aux autres, il propose le choix du support, à savoir l'image unique qu'est le daguerréotype ou l'image sur papier, reproductible, qu'est le calotype $^{12}$. Le travail de Le Blondel en fait l'une des figures emblématiques de la photographie professionnelle régionale. Sa renommée repose notamment sur son exploitation des calotypes, savoir-faire qu'il a acquis auprès de Louis-Désiré BlanquartÉvrard.

Au début des années 1850, de nouveaux ateliers ouvrent et s'adaptent au fur et à mesure à l'apparition de nouvelles techniques plus efficaces encore. L'ère de la photographie industrielle commence. En 1851, Blanquart-Évrard ouvre son imprimerie photographique dans la banlieue de Lille. Répondant aux besoins, à la fois des photographes et des éditeurs, il produit à moindre coût, rapidement, grâce à des procédés améliorés et amène ainsi la photographie dans le milieu industriel de l'époque.

Éditeur, Blanquart-Évrard supervise les tirages, leur montage au sein d'albums aux thématiques spécifiques et produit aussi des images pour d'autres tenants de l'industrie du livre. Ces derniers ont très tôt compris l'apport du lillois, et notamment que la combinaison d'un laboratoire de tirages et d'une imprimerie photographique constituaient une étape dans un processus encore en devenir. Précurseur, Blanquart-Évrard, fit bientôt des émules à Paris ${ }^{13}$.

Blanquart-Évrard introduit ainsi la photographie dans une nouvelle sphère et s'impose comme référence dans le milieu. Quatre ans plus tard, l'établissement ferme ses portes avec une production de plus 100000 épreuves et la diffusion de 24 albums. Les secrets de fonctionnement seront gardés jusqu'en $1862^{14}$.

Parallèlement à la photographie professionnelle, une autre forme se développe, celle des amateurs. Dès 1842, des cours de daguerréotypie sont organisés, notamment dans la ville de Valenciennes. Ainsi, les années 1850 voient la photographie se développer autant dans le domaine professionnel que dans celui des amateurs. Elle connaît une expansion géographique sur tout le territoire de la région. De nombreux studios de photographes voient le jour. Au fil des années, les commerces se transmettent de père en fils. C'est ainsi que certaines familles deviennent des noms incontournables dans le milieu, tels que Cayez à Lille, ou encore Delsart à Valenciennes $^{15}$.

11. Alphonse Le Blondel est né à Caen en 1814 et meurt à Lille en 1875. Peintre de formation, il s'installe comme portraitiste dans la région.

12. Évelyne-Dorothée Allemand, Katia Baudin, Damien Camus, et al., 160 ans de photographie..., ibid., p. 12.

13. Voir Jean-Claude Gautrand, Alain Busine, Blanquart-Évrard, Douchy-les-Mines, Centre Régional de la Photographie Nord-Pas-de-Calais, 1999, 177 p.

14. Voir Jean-Claude Gautrand, Alain Busine, Blanquart-Évrard, ibid.

15. Évelyne-Dorothée Allemand, Katia Baudin, Damien Camus, et al., 160 ans de photographie..., op. cit., p. 13. 
Cette transmission intergénérationnelle revêt certes un caractère commercial, mais il y a également une prise de conscience de la valeur que détiennent les images du passé. Ce sont les années qui voient se mettre en place une politique de sauve$\operatorname{garde}^{16}$.

"Le patrimoine a explosé dans tous les sens. [...] Il s'est échappé du notariat et de l'artistique pour envahir tous les domaines dont il était précisément exclu : le vécu traditionnel, le contemporain encore en usage [...] ${ }^{17}$. Cette évolution est liée à l'éveil de la conscience patrimoniale. L'intérêt pour la sauvegarde et la conservation du patrimoine s'est développé tout au long du XIX ${ }^{\mathrm{e}}$ siècle et va s'ouvrir sur de nouvelles catégories durant le $\mathrm{XX}^{\mathrm{e}}$ siècle. Cet élargissement, d'un "patrimoine national " à un "patrimoine de type social et communautaire", amène ainsi la photographie, à la sortie de la Première Guerre mondiale, au rang de patrimoine.

Les photographes deviennent alors les «conservateurs " du patrimoine de leur temps. L'accumulation de la documentation photographique semble s'être beaucoup plus développée dans le Nord-Pas-de-Calais que dans les autres régions de France ${ }^{18}$ et ces précieuses archives ont permis de voir l'évolution de la représentation au cours du temps.

En photographie, la notion de collection est apparue peu de temps après la création du médium, ce qui a permis la préservation de nombreuses images dès le XIX ${ }^{\mathrm{e}}$ siècle. En 1851, la Société héliographique ${ }^{19}$ crée un album où chacun de ses membres est invité à y laisser l'une de ses créations réalisées avec le procédé dont il se sert, concept qui a été repris en 1854 par la Société française de photographie ${ }^{20}$. La collection de cette dernière s'enrichit ainsi des créations de l'époque grâce aux multiples dons, qui augmentent au cours des expositions organisées. Comme cela a été évoqué précédemment, c'est également l'époque où Blanquart-Évrard publie ses premiers albums.

De nombreuses collections privées se constituent et les institutions culturelles vont commencer timidement à se tourner vers cette question de la collection photographique. Parallèlement, les collectionneurs privés ont rassemblé d'imposants corpus d'images, permettant non seulement de mieux faire connaître la photographie par le biais de publications qu'ils rédigeaient, mais surtout de préserver une consi-

16. La Révolution française (1789-1799) fut un temps de destruction, de vandalisme. C'est précisément parce qu'il faut sauver ce qui va être détruit, que naît cette notion de patrimoine. Ce sont les années 1830-1860 qui voient se mettre en place une politique de sauvegarde.

17. Voir Pierre Nora, Les lieux de mémoire, vol. 1, Paris, Quarto-Gallimard, 1997, 1664 p.

18. Évelyne-Dorothée Allemand, Katia Baudin, Damien Camus, et al., 160 ans de photographie..., op. cit., p. 13.

19. C'est en 1851 que la Société héliographique voit le jour. Parmi ses membres fondateurs se trouvent cinq photographes qui vont participer à l'inventaire des monuments historiques en photographiant ces édifices à travers toute la France. Il s'agit de Gustave Le Gray, Mestral, Édouard Baldus, Hippolyte Bayard et Henri Le Secq.

20. La Société française de photographie (SFP) est une association créée en 1854 par un groupe d'amateurs, de scientifiques et d'artistes. Elle a été reconnue d'utilité publique en 1892. La SFP possède l'une d'une des plus importantes collections patrimoniales privées consacrées à la photographie. 
dérable quantité de photographies qui, sans eux, étaient vouées à une disparition certaine. Conscient de la valeur de ces fonds, ce sera souvent vers les collectionneurs privés que se tournent les institutions culturelles pour récupérer de grands ensembles.

\section{Un rôle nouveau pour le médium photographique}

La fin du XIX ${ }^{\mathrm{e}}$ siècle marque un tournant pour la photographie. Les procédés se sont simplifiés et ouvrent alors le médium à un plus large public. Le réseau amateur se développe et de nombreuses sociétés voient le jour dans la région, comme par exemple la Société photographique de Valenciennes créée en 1892. Le rayonnement de leurs activités reste local, mais ces sociétés deviennent des centres de réflexion et de recherche sur la photographie et sont un élément moteur dans la création photographique régionale. Toutefois, la Première Guerre mondiale aura raison de bon nombre d'entre elles.

La période d'après-guerre aura certes des impacts "négatifs " dans le développement de la production photographique, que ce soit au niveau professionnel ou amateur, mais apportera également une vision nouvelle sur le médium en lui donnant une valeur patrimoniale.

Puisque le Nord et le Pas-de-Calais sont totalement dévastés à la sortie de ce terrible conflit, il faut penser à la reconstruction complète de la région (ill. 1 et 2). L'étendue des destructions est considérable :

Dans le Pas-de-Calais, 279 communes ont été affectées par les combats ; parmi elles, 167 sont totalement anéanties ( $1^{\text {er }}$ rang national), 57 détruites à plus de $50 \%$; dans le Nord, 220 communes ont connu des combats, 18 ont été totalement rasées, 65 détruites à plus de $50 \%$. Dans le Pas-de-Calais, on recense 102440 immeubles touchés par le conflit, [...] 526000 personnes sont sans abri (près de la moitié de la population d'avant-guerre); dans le Nord, 307300 immeubles ont été touchés [...]. Plusieurs villes ont été littéralement rayées de la carte; Bailleul, Lens, Liévin, Bapaume sont détruites à $100 \%, 85 \%$ des maisons d'Armentières doivent être reconstruites, Arras est atteinte à $75 \%$ [...], Béthune à $50 \%$; les destructions sont aussi importantes à Cambrai, à Douai, à Valenciennes. Lens et Liévin présentent la particularité d'avoir été entièrement arasées au niveau des fondations ${ }^{21}$.

La reconstruction des villes sinistrées s'achève au début des années 30. Toutefois, elle ne fut pas abordée sur l'ensemble du territoire de la même manière en matière de choix urbanistique. En effet, l'enjeu est de reconstruire rapidement les bâtisses d'avant-guerre en même temps qu'émerge une préoccupation de modernisation des villes.

21. Yves Le Maner, La Grande Guerre dans le Nord et le Pas-de-Calais : 1914-1918, Lille, La Voix, 2014, p. 370 . 


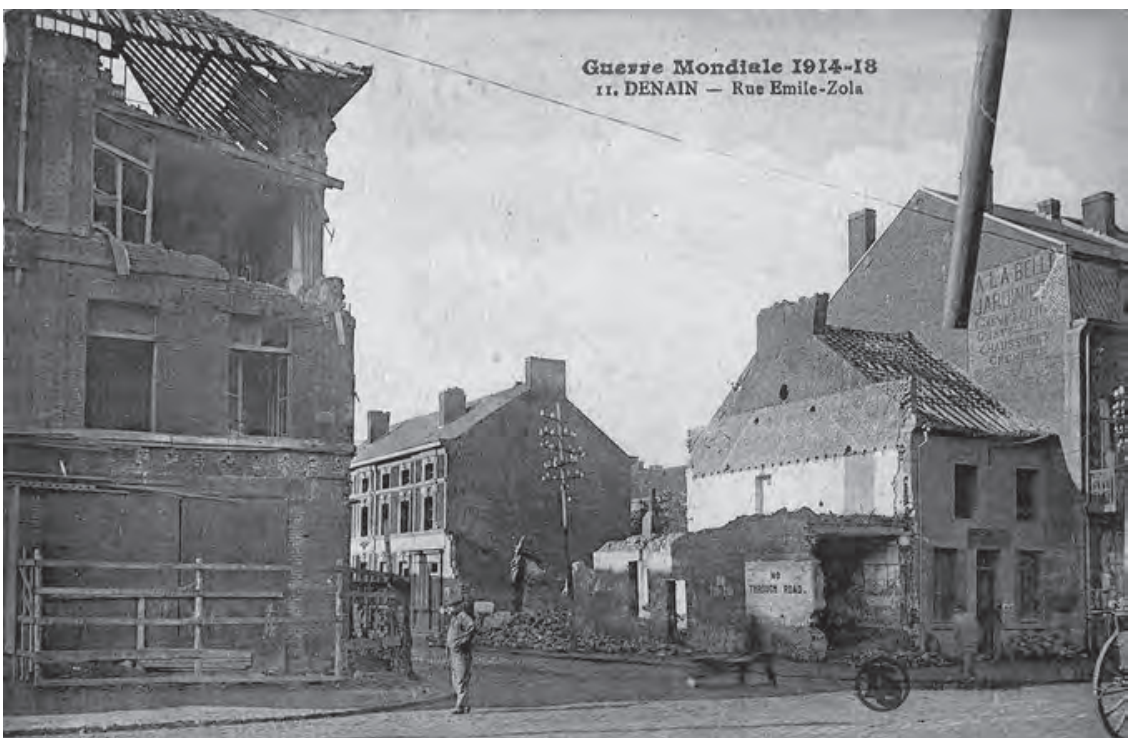

Ill. 1 : Denain. Rue Emile-Zola. Guerre Mondiale 1914-1918, Carte postale, $9 \times 14 \mathrm{~cm}$, Musée d'archéologie et d'histoire locale, Denain, $\mathrm{n}^{\circ}$ inventaire 03.9.20b. (c) CAPH - Germain Hirselj.

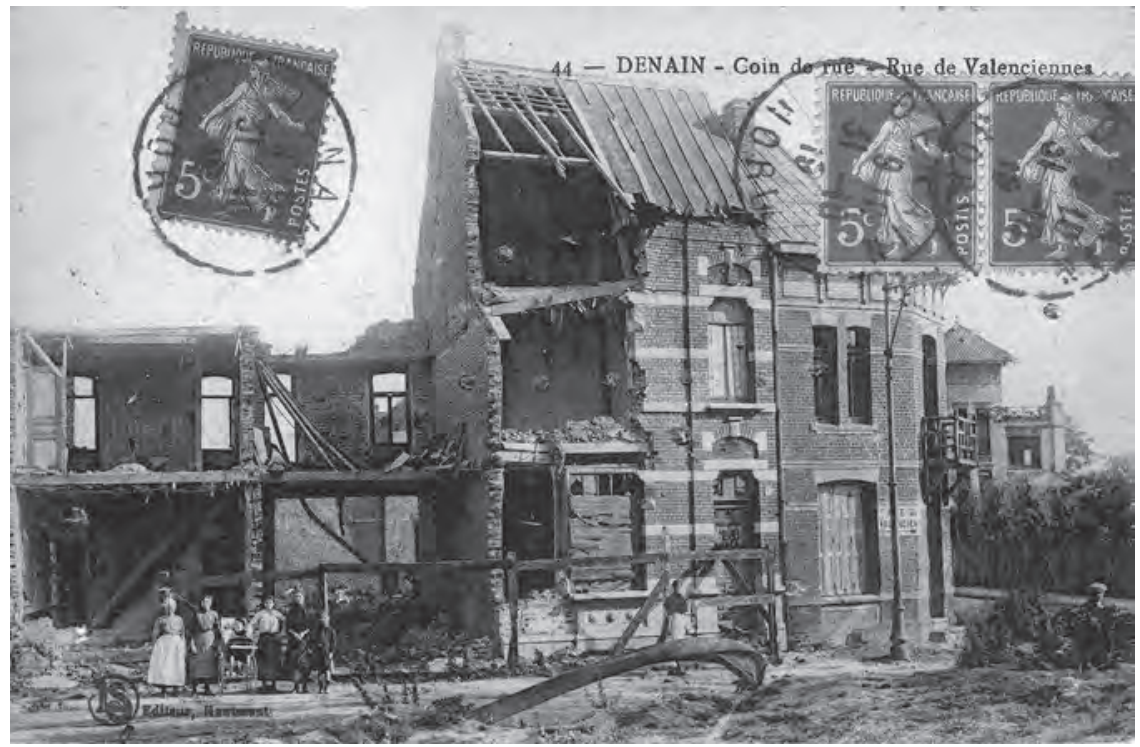

Ill. 2: Denain. Coin de rue - rue de Valenciennes, circa 1918, Carte postale, $9 \times 14 \mathrm{~cm}$, Musée d'archéologie et d'histoire locale, Denain, $n^{\circ}$ inventaire 96.9.18. () CAPH - Germain Hirselj. 
Certaines ont pris le parti de reconstruire à l'identique afin de retrouver leur identité et d'échapper au traumatisme de la Grande Guerre. Ce fut notamment le cas pour la ville d'Arras, l'une des plus touchées au cours de ce conflit. Lorsque l'architecte en chef des monuments historiques chargé du Pas-de-Calais, Pierre Paquet ${ }^{22}$ s'est vu confier la reconstruction de l'hôtel de ville et du beffroi d'Arras, symboles historiques de la ville, il n'avait aucun relevé précis des deux monuments détruits. Il dut mener un long travail pour réunir un considérable ensemble photographique ${ }^{23}$ afin de retrouver tous les détails de l'architecture des deux bâtiments ${ }^{24}$. Les photographies collectées représentaient l'unique trace des édifices disparus.

À l'instar d'Arras, la ville de Valenciennes ne fut pas épargnée et retrouva, notamment, à la sortie de la guerre, sa gare dans un piteux état. Elle fut reconstruite à l'identique ${ }^{25}$, comme bon nombre d'édifices dans la région, en s'appuyant sur les divers fonds photographiques répartis sur le territoire.

Toutefois, certaines villes ne s'engagèrent pas dans la reconstruction à l'identique, souvent contraignante et coûteuse. C'est ainsi que des villes ayant subi des dégâts considérables, comme Bailleul, saisirent l'opportunité d'une reconstruction totale permettant la modernisation des bâtiments et des rues. La reconstruction de Bailleul fut confiée à l'architecte Louis-Marie Cordonnier ${ }^{26}$, qui allia modernité et inspiration régionaliste. Le traitement des symboles de la ville, l'hôtel de ville et le beffroi, souligne l'inspiration flamande.

Dans ces villes qui ne furent pas reconstruites à l'identique, la photographie devient alors le témoin de ce qui a disparu. Qu'elles servent de base même à la reconstruction ou non, les photographies d'avant-guerre permettent de visualiser le patrimoine détruit. Ces photographies constituent un témoignage d'un territoire à un moment de son histoire et, à ce titre, elles se hissent au rang de patrimoine.

La photographie est un patrimoine, qui reflète l'ensemble des us et coutumes de la société, son vécu et son histoire. Elle représente une mémoire collective. En nous rappelant à nos souvenirs, la photographie devient un "lieu de mémoire ». Un objet, comme l'explique Pierre Nora, devient « lieu de mémoire » quand il échappe à l'oubli, par exemple avec l'apposition de plaques commémoratives et quand une collectivité le réinvestit de son affect et de ses émotions. Il nous explique également qu' "un lieu de mémoire dans tous les sens du mot va de l'objet le plus matériel et concret, éventuellement géographiquement situé, à l'objet le plus abstrait et intellectuellement construit ${ }^{27}$. La photographie est tout cela.

22. Pierre Paquet (1875-1959) est un architecte des monuments historiques qui s'est vu confier la reconstruction des monuments détruits dans les zones sinistrées du Nord et du Pas-de-Calais.

23. Il a mené sa collecte au sein des collections publiques mais également auprès de collectionneurs privés.

24. Voir : Conseil général du Pas-de-Calais, sous la direction de Patrice Marcilloux, Images de la reconstruction: Arras 1918-1934, Dainville, Archives départementales du Pas-de-Calais, 1997, 127 p.

25. À l'exception de la grande marquise couvrant les quais.

26. Louis-Marie Cordonnier (1854-1940) est un architecte français. Suite à la Première Guerre mondiale, il s'est vu confier la reconstruction des villes de Bailleul et Armentières.

27. Pierre Nora, Les lieux de mémoire, op. cit., p. 28. 
Un lien entre présent et passé se crée, entre réel et imaginaire. Il y a une puissance évocatrice dans la photographie. Elle nous touche, nous fait rêver, nous émerveille, mais elle peut aussi bouleverser. Elle est symbole de notre mémoire, de notre identité. A la sortie de la Grande Guerre, la photographie se hisse au rang de nouveau patrimoine, dans ce que Pierre Nora appelle "l'explosion du patrimoine".

Convoquant le souvenir, la photographie est la totalité manquante qui se dessine dans les contours de ce qui reste, une interaction entre présence et absence. Ainsi, la photographie nous conduit sur la scène d'un théâtre de la mémoire.

Mémoire et transmission, ces photographies d'avant-guerre parlent d'un passé, elles narrent une histoire. Patrimoniales, elles représentent l'identité d'une région, sa mémoire.

Angéline NisON

Chargée des collections au Centre régional de la photographie Hauts-de-France 